\title{
Construção e Validação da Tabela Nacional de Funcionalidade para as Doenças Crónicas
}

\section{Development and Validation of the National Functionality Table for Chronic Diseases}

\author{
Carla S. PEREIRA $\rrbracket^{1}$, Jaime BRANCO ${ }^{2,3}$, Manuel LOPES ${ }^{4}$, Ana ESCOVAL ${ }^{1}$, Paulo NOGUEIRA ${ }^{5,6}$, Alexandre DINIZ $^{6}$, \\ Fernando GUERRA ${ }^{7}$, Anabela COELHO 6 \\ Acta Med Port 2016 Feb;29(2):114-122 - http://dx.doi.org/10.20344/amp.6692
}

\section{RESUMO}

Introdução: A avaliação sistemática e registo da funcionalidade de pessoas adultas com doença crónica permite horizontalizar políticas de saúde, sociais e emprego de acordo com a funcionalidade; dotar os profissionais de saúde e sociais de um instrumento de recolha de informação, que complemente os registos de doença; medindo os ganhos de funcionalidade. O objetivo de estudo foi desenvolver uma Tabela Nacional de Funcionalidade para adultos em idade ativa com doença crónica, de acordo com a Classificação Internacional de Funcionalidade, Incapacidade e Saúde da Organização Mundial de Saúde.

Material e Métodos: Recorremos a métodos quantitativos e qualitativos; revisão da literatura (17 artigos), grupo focal (nove peritos), painel de Delphi (16 peritos) e estudo exploratório (309 pessoas com doença crónica).

Resultados: Na revisão da literatura, foram identificadas 67 atividades limitadas na população em estudo das quais foram selecionadas 40 atividades pelo grupo focal e 38 validadas pelo painel de Delphi.

Discussão: Para testar as propriedades psicométricas comparamos o valor médio de todos os coeficientes possíveis do tipo consistência interna (split-half). Na análise da discriminação dos níveis de funcionalidade em amostras diferentes, verificou-se a igualdade de variâncias pelo teste de Levene e a igualdade de média por recurso ao teste $t$. De acordo com a observação e análise do coeficiente a de Cronbach, verificou-se que a Tabela Nacional de Funcionalidade proposta apresenta bons níveis de fiabilidade. Na análise de componentes principais, identificaram-se cinco dimensões.

Conclusão: A referida tabela tem características psicométricas apropriadas no que diz respeito à consistência, fiabilidade e validade interna.

Palavras-chave: Adulto; Classificação Internacional de Funcionalidade Incapacidade e Saúde; Doença Crónica; Portugal.

\section{ABSTRACT}

Introduction: Systematic evaluation and registration of adults functionality with chronic diseases is relevant, because it allows: 'flattening' health, social and employment policies, according to the person's functionality with chronic diseases, providing health and social professionals with a data collection, which complements disease records, and finally, measuring functionality gains. The objective of the study was to develop a National Functionality Table for active age adults with chronic disease, according to the International Classification of Functioning, Disability and Health of the World Health Organization.

Material and Methods: Quantitative and qualitative methods were used; literature review (17 articles), focal group (nine experts), Delphi panel (16 experts) and exploratory study (309 persons with chronic diseases).

Results: The literature review identified 67 limited activities in the study population, from which 40 activities were selected by the focal group and 38 activities were validated by the Delphi panel.

Discussion: In order to test the psychometric properties we have compared the average value of all possible coefficients of internal consistency type (split-half). When analyzing the discrimination of functional levels in different samples, equality of variances was verified, using the Levene test as well as the " $t$ " test. According to the observation and analysis of a Cronbach coefficient we have found that the National Functionality Table shows good reliability levels. From the main component analysis, five dimensions were identified. Conclusion: the National Functionality Table has appropriate psychometric characteristics in respect to its consistency, reliability and internal validity.

Keywords: Adult; Chronic Disease; Disability and Health; International Classification of Functioning; Portugal.

\section{INTRODUÇÃo}

As doenças crónicas, como doenças cardíacas, acidente vascular cerebral, cancro, doenças respiratórias crónicas e diabetes, são a principal causa de mortalidade no mundo, representando $60 \%$ das causas de morte. ${ }^{1}$ Os custos associados às doenças crónicas incluem, gastos monetários com os cuidados de saúde e, toda a despesa invisível associada à doença crónica, como sejam a diminuição da esperança média de vida, a condição de incapacidade e participação diminuída de alguns grupos mais vulneráveis, a redução dos dias de trabalho, o absentismo, o desemprego

1. Centro de Investigação em Saúde Pública. Escola Nacional de Saúde Pública. Universidade Nova de Lisboa. Lisboa. Portugal.

2. Centro de Estudo de Doença Crónica. NOVA Medical School. Universidade NOVA de Lisboa. Lisboa. Portugal.

3. Serviço de Reumatologia. Hospital Egas Moniz. Centro Hospitalar Lisboa Ocidental. Lisboa. Portugal.

4. Escola Superior de Enfermagem S. João de Deus. Universidade de Évora. Évora. Portugal.

5. Instituto de Medicina Preventiva e Saúde Publica. Faculdade de Medicina. Universidade de Lisboa. Lisboa. Portugal.

6. Departamento da Qualidade na Saúde. Direção-Geral da Saúde. Lisboa. Portugal.

7. Faculdade de Medicina. Universidade de Lisboa. Lisboa. Portugal.

$\square$ Autor correspondente: Carla Sandra Martins Pereira: carlasandrapereira@gmail.com

Recebido: 09 de junho de 2015 - Aceite: 29 de setembro de 2015 | Copyright @ Ordem dos Médicos 2016 
e a diminuição da qualidade de vida. Segundo a Organização Mundial de Saúde (OMS), o aumento das doenças crónicas é uma 'epidemia invisível' e uma causa subestimada de pobreza, que impede o desenvolvimento económico de muitos países. ${ }^{2}$

A implementação do registo sistemático da funcionalidade do adulto permite, ao longo do tempo, medir a evolução da funcionalidade, como indicador de saúde, contribuindo, assim, para uma mais adequada e racional alocação de recursos, monitorização de resultados das intervenções de saúde e sociais, assim como para uma melhoria da equidade na atribuição de benefícios fiscais e prestações sociais. ${ }^{3}$ Foi objetivo deste estudo, desenvolver uma tabela nacional de funcionalidade para adultos com doença crónica, de acordo com a Classificação Internacional de Funcionalidade, Incapacidade e Saúde (CIF) da Organização Mundial de Saúde.

Os objetivos específicos da $\mathrm{CIF}^{4}$ (DGS, 2004) são proporcionar uma base científica para a compreensão e estudo da saúde e dos estados relacionados com ela; estabelecer uma linguagem comum para descrever a saúde e os estados relacionados com ela; melhorar a comunicação entre os distintos utilizadores (profissionais da saúde, investigadores, gestores de políticas sanitárias e a população geral), incluindo os adultos com incapacidades; permitir a comparação de dados entre países, entidades sanitárias, serviços, em diferentes momentos e ao longo do tempo; proporcionar um esquema de codificação sistematizado para ser aplicado nos sistemas de informação sanitária. ${ }^{4}$

Em Portugal identificaram-se algumas barreiras ao processo de implementação da $\mathrm{CIF}^{5}$ a saber: desconhecimento da classificação e do seu sistema de qualificadores; ausência de sistema métrico de quantificação; tempo de aplicação; pertinência da aplicação da classificação em contexto específico; falta de conhecimento generalizado sobre a CIF; falta de mecanismos de cooperação entre diferentes agentes que podem utilizar a CIF e ausência de plano estratégico para a implementação desta classificação, no contexto do Sistema de Saúde. Como o objetivo de quantificar a funcionalidade de forma consistente e rápida, através de uma nomenclatura/referencial comum, baseado especificamente nos componentes da CIF, construímos uma Tabela Nacional da Funcionalidade (TNF), centrada no componente 'Atividades e Participação'. Este componente reúne os domínios da funcionalidade, tanto na perspetiva individual, como na perspetiva social.

Neste estudo, para classificar a lista de 'Atividades e Participação', foi considerado o qualificador desempenho, descrito como o que o indivíduo faz no seu habitual ambiente de vida. Este qualificador também pode ser entendido como o 'envolvimento numa situação de vida', ou 'a experiência vivida' das pessoas no seu contexto de vida. ${ }^{4}$

Para complementar a avaliação considerámos, igualmente, a identificação dos 'Fatores Ambientais' por os mesmos poderem influenciar, de forma positiva ou negativa, o desempenho das atividades em análise. Estes fatores devem ser codificados de acordo com a situação que está a ser descrita (ex. o fator ambiental: rampas com piso liso pode ser codificado como um fator facilitador da atividade 'deslocar-se', no caso de uma pessoa em cadeira de rodas ou como uma barreira à atividade no caso de um invisual). ${ }^{4}$

\section{MATERIAL E MÉTODOS}

Para alcançar o objetivo de estudo recorreu-se a uma metodologia mista (métodos quantitativos e qualitativos), composta por diferentes procedimentos metodológicos que podem ser agrupados em dois momentos de estudos distintos: a) Desenho da TNF para as doenças crónicas; b) Validação nacional da TNF

a) Com o objetivo específico de identificar as atividades e áreas de participação mais limitadas na população adulta com doença crónica, efetuámos uma revisão da literatura, tomando como evidência os diversos Comprehensive ICF Core Set. Para o objetivo específico; desenhar o questionário de suporte da TNF, recorremos à metodologia Grupo Focal com 10 peritos na CIF e doenças crónicas.

b) Para a validação de conteúdo, realizamos a metodologia Painel de Delphi constituído por 13 médicos peritos em sistemas de verificação de incapacidade. Para a validação de construção ou teórica da TNF optámos por realizar metodologia exploratória transversal, com um pré-teste aplicado em sete Agrupamentos de Centros de Saúde (ACES) de Portugal Continental, três associações de doentes, Instituto Português de Oncologia de Lisboa Francisco Gentil e Instituto de Oftalmologia Dr. Gama Pinto. A fiabilidade interna foi testada por recurso á análise do coeficiente alfa de Cronbach e a consistência interna pela análise do split-half. Optámos ainda por realizar discriminação dos níveis de funcionalidade em amostras diferentes recorrendo ao teste de homogeneidade de variâncias de Levene e teste $t$ de igualdade de médias.

A população de referência, para a construção desta tabela, foi, adultos com doença crónica e idade compreendida entre os 18 e os 64 anos. A população com idade igual ou superior a 65 anos foi excluída, em virtude de já estarem a ser desenvolvidos estudos a nível nacional, que caracterizam a funcionalidade desta população. ${ }^{2} \mathrm{~A}$ população com idade inferior a 18 anos foi excluída, uma vez que o desempenho funcional previsto para crianças e jovens difere do dos adultos.

Para os distintos métodos de recolha de informação foram identificadas diversas amostras, como se observa na Tabela 1.

\section{RESULTADOS \\ Desenho da TNF para as doenças crónicas}

Para identificar as atividades e áreas de participação mais limitadas na população adulta com doença crónica foi realizada uma revisão da literatura. Os Comprehensive ICF Core Set publicados, internacionalmente, que foram considerados para revisão foram os seguintes:

1. Comprehensive ICF Core Set for Breast Cancer6;

2. Comprehensive ICF Core Set for Chronic Ischemic Heart Disease?; 
Tabela 1 - Amostras definidas para os diferentes objetivos específicos

\begin{tabular}{|c|c|c|c|}
\hline $\begin{array}{l}\text { Método de } \\
\text { recolha de } \\
\text { informação }\end{array}$ & Critério de exclusão da amostra & Critérios de inclusão na amostra & Amostra \\
\hline 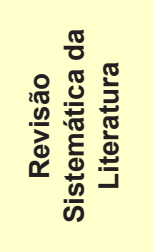 & $\begin{array}{l}\text { Artigos com metodologia pouco clara, } \\
\text { repetidos nas duas bases de dados, } \\
\text { com data anterior ao ano } 2001 \text { e todos } \\
\text { aqueles sem relação com o objeto de } \\
\text { estudo. }\end{array}$ & $\begin{array}{l}\text { - Artigos científicos publicados em texto integral, entre } \\
2000 / 01 / 01 \text { e } 2011 / 01 / 31 \text { por recursos à base de da- } \\
\text { dos eletrónica EBSCO; } \\
\text { - Artigos que descrevem as categorias ou códigos de } 1^{\circ} \text { e } \\
2^{\circ} \text { nível da CIF, utilizados para caracterizar a funcio- } \\
\text { nalidade da população com doença crónica. }\end{array}$ & 17 Artigos \\
\hline 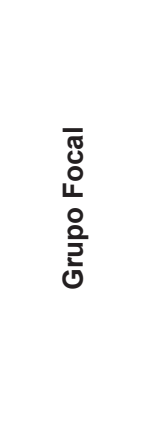 & $\begin{array}{l}\text { Profissionais de saúde com menos } \\
\text { de } 10 \text { anos de experiência na área da } \\
\text { doença crónica e da CIF }\end{array}$ & $\begin{array}{l}\text { - Profissionais com experiência de investigação em popu- } \\
\text { lação com doença crónica; } \\
\text { - Conhecimento da CIF; } \\
\text { - Experiência profissional na área da gestão ou direção } \\
\text { de serviços; } \\
\text { - Experiência na atividade de docência; } \\
\text { - Publicação ou apresentação de trabalhos nas áreas da } \\
\text { doença crónica ou CIF. }\end{array}$ & 9 Peritos \\
\hline 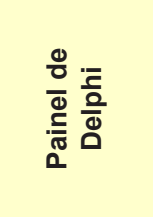 & $\begin{array}{l}\text { Profissionais de saúde com experiência } \\
\text { inferior a } 7 \text { anos na atribuição de } \\
\text { certificados de incapacidade }\end{array}$ & $\begin{array}{l}\text { - Profissionais de saúde com mais de } 7 \text { anos de expe- } \\
\text { riência em Sistema de Verificação de Incapacidade } \\
\text { identificados pelo Instituto de Segurança Social, IP } \\
\text { em todo o país; } \\
\text { - Conhecimento da tabela nacional de incapacidade. }\end{array}$ & 16 Peritos \\
\hline 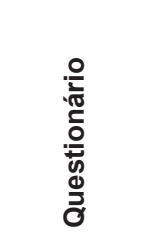 & $\begin{array}{l}\text { Homens e mulheres com menos de } 18 \\
\text { e mais de } 65 \text { anos em } 31 \text { de Janeiro } \\
\text { de } 2011 \text {, sem estarem registados nos } \\
\text { sistemas de saúde, e sem doença } \\
\text { crónica declarada. }\end{array}$ & $\begin{array}{l}\text { - Homens e mulheres com mais } 18 \text { anos e menos de } 65 \\
\text { anos a } 31 \text { de janeiro (inclusive) de 2011, registados } \\
\text { nas bases de dados dos sistemas nacionais de saúde } \\
\text { e nas associações de doentes, com pelo menos uma } \\
\text { doença crónica. }\end{array}$ & 309 Pessoas \\
\hline
\end{tabular}

3. Comprehensive ICF Core Set for Chronic Widespread Pain $^{8}$;

4. Comprehensive ICF Core Set for Depression ${ }^{9}$;

5. Comprehensive ICF Core Set for Diabetes Mellitus ${ }^{10,11}$;

6. Comprehensive ICF Core Set for Low Back Pain ${ }^{12}$;

7. Comprehensive ICF Core Set for Obesity ${ }^{13}$;

8. Comprehensive ICF Core Set for Obstructive Pulmonary Diseases ${ }^{14}$;

9. Comprehensive ICF Core Set for Osteoarthritis ${ }^{15}$;

10. Comprehensive ICF Core Set for Osteoporosis ${ }^{16}$;

11. Comprehensive ICF Core Set for Rheumatoid Arthritis ${ }^{17}$;

12. Comprehensive ICF Core Set for Stroke ${ }^{18}$;

13. ICF CHECKLIST.

Para otimizar a análise da informação disponível foram analisadas as frequências dos códigos da CIF de segundo nível, na dimensão das atividades e a participação (Tabela 2). Foram identificados 67 'atividades/participação' que serviram de base de trabalho para o procedimento metodológico seguinte, de seriação dos itens através do grupo focal que teve por objetivos específicos: Adaptar ao contexto nacional a lista de atividades e participações identificadas; Adaptar o sistema de qualificadores da CIF ao contexto nacional; Redação do manual para aplicação da TNF para a população adulta com doença crónica.
Para o grupo focal foram convidados dez peritos, que reuniam os critérios de inclusão definidos, de acordo com o sugerido na literatura, a saber:

1. Experiência profissional no setor da Saúde;

2. Experiência profissional com população portadora de doença crónica;

3. Conhecimento e manuseio de classificações da OMS;

4. Conhecimento da CIF;

5. Experiência de participação em grupo de reflexão;

6. Adesão ao código de ética do departamento da qualidade na Saúde da Direção-Geral da Saúde.

O grupo focal garantiu o cumprimento dos princípios éticos e deontológicos nas diferentes fases do estudo, tendo em consideração o Artigo $35 .^{\circ}$ da Constituição da República Portuguesa e o Artigo $15 .^{\circ}$ da Lei n. ${ }^{\circ} 67 / 98$ de 26 de outubro.

Os resultados obtidos por grupo focal foram:

- Seleção dos itens, resultantes da revisão da literatura, que melhor caracterizassem a funcionalidade da população portuguesa, adulta em idade laboral com doença crónica;

- Adaptação semântica no sistema métrico de qualificação, tendo por referência os qualificadores da CIF;

- Desenho do manual de procedimentos necessários 
para a recolha de dados e apresentação à amostra e às entidades que recolhem os dados;

- Redação do guião de perguntas para aplicação da TNF;

- Construção do manual de aplicação da tabela.

\section{Validação da TNF}

Com o objetivo de validar a consistência interna da TNF, foram identificados 13 médicos peritos em sistemas de verificação de incapacidade, em nove distritos do ter- ritório Português, (Aveiro, Castelo Branco, Évora, Lisboa, Porto, Setúbal, Vila Real, Região Autónoma dos Açores e Região Autónoma da Madeira), garantindo a representatividade nacional. Os critérios de caracterização dos peritos que participaram na validação da consistência interna da tabela, foram: conhecimento acerca da aplicação da CIF; habilitações académicas (especialidade médica); tempo de experiência na atribuição de certificados de incapacidade: atividade de docência e/ou investigação nas áreas da

Tabela 2 - Frequências dos códigos da CIF de $2^{\circ}$ nível, na dimensão das atividades e a participação identificados na revisão da literatura

\begin{tabular}{|c|c|c|c|}
\hline Códigos CIF & $\begin{array}{l}\mathrm{N}^{\circ} \text { de } \\
\text { referências }\end{array}$ & Códigos CIF & $\begin{array}{l}\mathrm{N}^{\circ} \text { de } \\
\text { referências }\end{array}$ \\
\hline D110 Observar & 1 & D465 Deslocar-se utilizando equipamentos & 3 \\
\hline D115 Escutar & 2 & D470 Utilizar transportes & 4 \\
\hline D140 Aprender a ler & 0 & D475 Conduzir & 4 \\
\hline D145 Aprender a escrever & 0 & D510 Lavar-se & 8 \\
\hline D150 Aprender a calcular & 0 & D520 Cuidar de partes do corpo & 5 \\
\hline D155 Adquirir competências & 1 & D530 Toalete & 6 \\
\hline D160 Concentrar a atenção & 1 & D540 Vestir & 8 \\
\hline D166 Ler & 3 & D550 Comer & 4 \\
\hline D170 Escreve & 1 & D560 Beber & 3 \\
\hline D172 Calcular & 1 & D570 Cuidar da saúde & 10 \\
\hline D175 Revolver problemas & 3 & D620 Adquirir bens e serviços & 12 \\
\hline D177 Tomar decisões & 3 & D630 Preparar refeições & 8 \\
\hline D210 Realizar uma tarefa & & D640 Fazer trabalhos domésticos & 10 \\
\hline D220 Realizar múltiplas tarefas & 2 & D650 Cuidar dos objetos de casa e ajudar os outros & 5 \\
\hline D230 Realizar a rotina diária & 3 & D660 Dar assistência a terceiros & 8 \\
\hline $\begin{array}{l}\text { D240 Lidar com o stress e outras exigências } \\
\text { psicológicas }\end{array}$ & 7 & D710 Interações interpessoais básicas & 4 \\
\hline $\begin{array}{l}\text { D310 Comunicar com - Receber - Mensagens } \\
\text { faladas }\end{array}$ & 8 & D720 Interações interpessoais complexas & 2 \\
\hline D325 Comunicar e receber mensagens escritas & 1 & D730 Relacionamento com estranhos & 1 \\
\hline $\begin{array}{l}\text { D315 Comunicar com - Receber - Mensagens } \\
\text { não-verbais }\end{array}$ & 2 & D740 Relacionamentos formais & 1 \\
\hline D330 Falar & & D750 Relacionamentos sociais informais & 5 \\
\hline D335 Produzir mensagens não-verbais & 2 & D760 Relações familiares & 9 \\
\hline D345 Escrever mensagens & 1 & D770 Relacionamentos íntimos & 12 \\
\hline D350 Conversação & 2 & D810 Educação informal & 1 \\
\hline D355 Discussão & 1 & D820 Educação escolar & 1 \\
\hline $\begin{array}{l}\text { D360 Utilização de dispositivos e técnicas de } \\
\text { comunicação }\end{array}$ & 2 & D830 Educação superior & 3 \\
\hline D410 Mudar a posição básica do corpo & 4 & D845 Formação profissional & 7 \\
\hline D415 Manter a posição do corpo & 3 & D850 Emprego remunerado & 9 \\
\hline D420 Auto transferência & 1 & D855 Emprego não remunerado & 2 \\
\hline D430 Levantar e carregar objetos & 4 & D859 Trabalho ou emprego, ou outro não específico & 2 \\
\hline D440 Motricidade fina & 3 & D860 Transações económicas básicas & 3 \\
\hline D445 utilização da mão e do braço & 1 & D865 Transações económicas complexas & 1 \\
\hline D449 Transportar, mover e manusear objetos & 2 & D870 Autossuficiência económica & 2 \\
\hline D450 Andar & 4 & D910 Vida em comunidade & 8 \\
\hline D455 Deslocar-se & 4 & D920 Recreação e lazer & 10 \\
\hline D460 Deslocar-se por diferentes locais & 3 & D950 Vida política e cidadania & 1 \\
\hline
\end{tabular}


deficiência e incapacidade.

Sendo a técnica de Delphi utilizada para a obtenção de consensos, realizaram-se duas rondas. ${ }^{19} \mathrm{Na}$ primeira, utilizaram-se questões com resposta 'fechada', com recurso a uma escala de concordância do tipo Likert.

Tabela 3 - Níveis de concordância obtidos por painel de Delphi

\begin{tabular}{|c|c|c|c|c|c|c|}
\hline \multirow{2}{*}{ Atividades/participação } & \multicolumn{2}{|c|}{$\begin{array}{l}\text { \% de concordância com } \\
\text { a inclusão da atividade/ } \\
\text { participação na tabela }\end{array}$} & \multicolumn{2}{|c|}{$\begin{array}{l}\text { \% de concordância com } \\
\text { pergunta 'tipo' proposta }\end{array}$} & \multicolumn{2}{|c|}{$\begin{array}{c}\text { \% de concordância com a } \\
\text { tradução semântica dos } \\
\text { qualificadores }\end{array}$} \\
\hline & $1^{\text {a }}$ Ronda & $2^{\text {a Ronda }}$ & $1^{\text {a }}$ Ronda & $2^{\text {a }}$ Ronda & $1^{\text {a }}$ Ronda & $2^{\text {a Ronda }}$ \\
\hline Ler & $80 \%$ & $87 \%$ & $74 \%$ & $93 \%$ & $83 \%$ & $87 \%$ \\
\hline Resolver problemas & $80 \%$ & $93 \%$ & $78 \%$ & $100 \%$ & $74 \%$ & $73 \%$ \\
\hline Realizar tarefas múltiplas & $85 \%$ & $87 \%$ & $74 \%$ & $87 \%$ & $75 \%$ & $87 \%$ \\
\hline Realizar a rotina & $88 \%$ & $93 \%$ & $77 \%$ & $100 \%$ & $82 \%$ & $87 \%$ \\
\hline Lidar com o stress e outras exigências psicológicas & $78 \%$ & $93 \%$ & $78 \%$ & $80 \%$ & $75 \%$ & $67 \%$ \\
\hline Falar & $86 \%$ & $100 \%$ & $85 \%$ & $100 \%$ & $80 \%$ & $93 \%$ \\
\hline Conversação & $86 \%$ & $93 \%$ & $77 \%$ & $100 \%$ & $80 \%$ & $93 \%$ \\
\hline Utilização de dispositivos de comunicação & $69 \%$ & $73 \%$ & $75 \%$ & $73 \%$ & $83 \%$ & $67 \%$ \\
\hline Mudar a posição do corpo & $86 \%$ & $100 \%$ & $82 \%$ & $93 \%$ & $89 \%$ & $93 \%$ \\
\hline Manter a posição do corpo & $86 \%$ & $100 \%$ & $78 \%$ & $93 \%$ & $85 \%$ & $93 \%$ \\
\hline Motricidade fina & $83 \%$ & $93 \%$ & $83 \%$ & $87 \%$ & $86 \%$ & $80 \%$ \\
\hline Utilização da mão e braço & $89 \%$ & $100 \%$ & $86 \%$ & $100 \%$ & $83 \%$ & $93 \%$ \\
\hline Transportar, mover e manusear objetos & $82 \%$ & $93 \%$ & $78 \%$ & $93 \%$ & $86 \%$ & $93 \%$ \\
\hline Andar & $91 \%$ & $100 \%$ & $80 \%$ & $93 \%$ & $85 \%$ & $80 \%$ \\
\hline Deslocar-se por diferentes locais & $83 \%$ & $87 \%$ & $82 \%$ & $93 \%$ & $83 \%$ & $80 \%$ \\
\hline Deslocar-se utilizando equipamentos & $75 \%$ & $100 \%$ & $75 \%$ & $87 \%$ & $86 \%$ & $73 \%$ \\
\hline Utilizar transporte, carro, autocarro, comboio & $78 \%$ & $93 \%$ & $77 \%$ & $93 \%$ & $83 \%$ & $93 \%$ \\
\hline Conduzir & $68 \%$ & $67 \%$ & $71 \%$ & $87 \%$ & $77 \%$ & $97 \%$ \\
\hline Lavar-se mãos e corpo & $91 \%$ & $100 \%$ & $86 \%$ & $100 \%$ & $88 \%$ & $93 \%$ \\
\hline Cuidar de partes do corpo & $91 \%$ & $100 \%$ & $83 \%$ & $100 \%$ & $91 \%$ & $93 \%$ \\
\hline Cuidados com o processo de excreção & $89 \%$ & $100 \%$ & $77 \%$ & $80 \%$ & $83 \%$ & $93 \%$ \\
\hline Vestir-se & $89 \%$ & $100 \%$ & $85 \%$ & $100 \%$ & $89 \%$ & $67 \%$ \\
\hline Alimentar-se - comer e beber & $89 \%$ & $100 \%$ & $82 \%$ & $100 \%$ & $88 \%$ & $80 \%$ \\
\hline Cuidar da saúde & $85 \%$ & $93 \%$ & $77 \%$ & $80 \%$ & $83 \%$ & $80 \%$ \\
\hline Adquirir bens e serviços (fazer compras, etc.) & $77 \%$ & $80 \%$ & $80 \%$ & $87 \%$ & $83 \%$ & $60 \%$ \\
\hline Preparar refeições & $71 \%$ & $93 \%$ & $78 \%$ & $93 \%$ & $75 \%$ & $93 \%$ \\
\hline Fazer trabalhos domésticos & $72 \%$ & $80 \%$ & $78 \%$ & $87 \%$ & $82 \%$ & $73 \%$ \\
\hline Ajudar os outros & $68 \%$ & $53 \%$ & $77 \%$ & $80 \%$ & $78 \%$ & $60 \%$ \\
\hline Interações interpessoais básicas & $78 \%$ & $93 \%$ & $77 \%$ & $93 \%$ & $78 \%$ & $93 \%$ \\
\hline Relacionamentos sociais informais & $75 \%$ & $67 \%$ & $72 \%$ & $87 \%$ & $77 \%$ & $73 \%$ \\
\hline Relações familiares & $78 \%$ & $93 \%$ & $82 \%$ & $93 \%$ & $82 \%$ & $87 \%$ \\
\hline Relacionamentos íntimos & $71 \%$ & $73 \%$ & $72 \%$ & $87 \%$ & $77 \%$ & $87 \%$ \\
\hline Formação profissional & $65 \%$ & $60 \%$ & $75 \%$ & $60 \%$ & $75 \%$ & $53 \%$ \\
\hline Educação de nível superior & $54 \%$ & $20 \%$ & $68 \%$ & $87 \%$ & $63 \%$ & $60 \%$ \\
\hline Obter, manter e sair do emprego & $78 \%$ & $87 \%$ & $78 \%$ & $80 \%$ & $78 \%$ & $87 \%$ \\
\hline Emprego remunerado & $77 \%$ & $80 \%$ & $71 \%$ & $87 \%$ & $80 \%$ & $80 \%$ \\
\hline Autossuficiência económica & $71 \%$ & $100 \%$ & $72 \%$ & $100 \%$ & $75 \%$ & $93 \%$ \\
\hline Transações económicas básicas & $78 \%$ & $87 \%$ & $72 \%$ & $93 \%$ & $80 \%$ & $73 \%$ \\
\hline Vida em comunidade & $78 \%$ & $87 \%$ & $78 \%$ & $93 \%$ & $85 \%$ & $80 \%$ \\
\hline Recreação e lazer & $72 \%$ & $73 \%$ & $77 \%$ & $93 \%$ & $80 \%$ & $73 \%$ \\
\hline
\end{tabular}

As questões foram:

a. Da lista de atividades e participação que se segue, concorda quanto à sua pertinência, para classificar a funcionalidade das pessoas adultas com doença crónica?

b. Concorda com as perguntas 'tipo', escritas no manual, 
para cada uma das atividades e participação identificadas, para classificar a funcionalidade das pessoas adultas com doença crónica?

c. Concorda com o exemplo de tradução semântica dos qualificadores apresentados no manual, para cada uma das atividades e participação identificadas para classificar a funcionalidade das pessoas com doença crónica? Os resultados desta fase indicaram que, com a linha de corte no grau de concordância superior a $50 \%$, não foi excluído nenhum dos itens da primeira para a segunda ronda.

A segunda ronda foi realizada 10 dias após a primeira. Quando interrogados com as mesmas questões, e após um período de reflexão de 15 dias, seguindo o mesmo nível de corte (concordância superior a 50\%) verifica-se que a atividade/participação 'educação de nível superior' obteve um grau de concordância inferior a $50 \%$ para inclusão desta atividade na TNF, sendo, por isso, excluída nos procedimentos de validação subsequentes. Relativamente às restantes 'atividades/participação' apenas as atividades 'conduzir', 'ajudar os outros', 'relacionamentos sociais informais' e 'formação profissional' obtiveram níveis de concordância inferiores a $70 \%$, contudo, optou-se por manter estas "atividades/participação" nos restantes processos de validação (Tabela 3).

Concluído o processo de validação de conteúdo da TNF de que resultou a lista final de atividades e participação a incluir na TNF, iniciou-se o processo de validação de construção, testada no pré-teste nacional, segundo uma metodologia exploratória.

Para aferir as propriedades psicométricas da TNF foi realizado um pré-teste a nível nacional, em ACES, com doentes sem certificado de incapacidade (CIT) atribuído e doentes com certificado de incapacidade atribuído.

A amostra foi aleatória, estratificada e por conglomerados. Os critérios de estratificação da amostra foram: distribuição proporcional da população geral pelas regiões de saúde, por sexo e grupo etário (18 - 34; 35 - 49; 50 - 64). Relativamente ao tamanho da amostra para um nível de confiança de $95 \%$, uma precisão absoluta de $1 \%$, o tamanho da amostra inicialmente preconizado foi de 1200 pessoas, o que permitiria uma precisão de intervalo de confiança para média numa escala a variar entre 0 e 100 pontos, inferior a um ponto ou um erro inferior a 0,5 pontos. Mas apenas responderam 309 indivíduos, o que confere uma precisão do intervalo de confiança de $95 \%$ - inferior a dois pontos ou um erro inferior a um ponto, ou seja apenas se conseguiu cerca de metade da precisão inicialmente desejada (Tabela 4).

As dimensões de estratificação da amostra por patologia crónica foram: diagnóstico confirmado das 12 patologias crónicas mais prevalentes (acidente vascular cerebral, artrite reumatóide, cancro da mama, depressão, diabetes mellitus, doença cardíaca isquémica crónica, doença oncológica, doença pulmonar obstrutiva crónica, dor generalizada crónica, espondilite anquilosante, lombalgia, obesidade, osteoartrose, osteoporose, paralisia cerebral); sexo; idade; possuir um CIT prorrogado no último ano; Sem nenhum CIT no último ano: registada em um dos 10 agrupamentos de centro de saúde aleatorizados/estratificados. Para robustecer o espectro de aplicação da tabela incluíram-se, ainda, doentes com patologia crónica mais incapacitante, tendo-se, para o efeito, recorrido ao Instituto Português de Oncologia de Lisboa, à Associação Nacional de Doentes com Artrite Reumatóide, à Associação Nacional de Espondilite Anquilosante, à Associação de Paralisia Cerebral de Lisboa e ao Instituto Gama Pinto.

O cálculo da amostra teve os seguintes pressupostos estatísticos: de acordo com a informação existente e assumindo que a escala a usar varia entre 0 e 100 pontos/ unidades, considera-se que, em teoria Gaussiana, 99,99\% destas pontuações se estendem ao longo de seis desvios-padrão. Considerando as classes definidas, a partir daquelas pontuações são esperadas algumas assimetrias (o que aponta para maior dispersão das pontuações). Deste modo, estima-se que o desvio-padrão da pontuação seja, no mínimo, 16,7 unidades, permitindo a assunção de 20 unidades para alojar alguma assimetria.

Sabendo que a missão dos ACES é garantir a prestação de cuidados de saúde primários à população de uma área geográfica determinada, definiram-se critérios de estratificação/aleatorização de 10 ACES com o objetivo de ter uma representação nacional da amostra onde se irá testar a TNF para as 12 doenças crónicas mais prevalentes. Para o efeito, realizou-se uma aleatorização de dois ACES por ARS, uma para a região litoral, outro para a região interior de cada ARS.

Os procedimentos realizados para a recolha de dados, foram: a) solicitar a cada ACES aleatorizado a nomeação de um elemento de ligação, responsável pela recolha e envio dos dados para o coordenador do projeto; b) construção de um formulário eletrónico, que ficou alojado no website da DGS; c) distribuição a todos os colaboradores do manual de utilização da plataforma e o guião de aplicação da tabela; d) aplicação da tabela durante o estudo exploratório (pré-teste nacional), em entrevista, dirigida por um profissional de saúde e registada em plataforma digital, construída para o efeito, alojada no website da DGS; e) atribuição de

Tabela 4 - Distribuição total de pessoas com doença crónica

\begin{tabular}{lrrrr}
\hline & N & \multicolumn{1}{c}{$\%$} & \% val. & \% acumulada \\
\hline Aveiro & 4 & 1,3 & 1,3 & 1,6 \\
Braga & 2 & 0,6 & 0,6 & 2,3 \\
Caldas da Rainha & 4 & 1,3 & 1,3 & 3,6 \\
Coimbra & 1 & 0,3 & 0,3 & 3,9 \\
Évora & 85 & 27,5 & 27,5 & 31,4 \\
Faro & 75 & 24,3 & 24,3 & 55,7 \\
Leiria & 1 & 0,3 & 0,3 & 56,0 \\
Lisboa & 79 & 25,6 & 25,6 & 81,6 \\
Portalegre & 1 & 0,3 & 0,3 & 81,9 \\
Santarém & 5 & 1,6 & 1,6 & 83,5 \\
Setúbal & 43 & 13,9 & 13,9 & 97,4 \\
Tomar & 8 & 2,6 & 2,6 & 100,0 \\
Total & $\mathbf{3 0 9}$ & $\mathbf{1 0 0 , 0}$ & $\mathbf{1 0 0 , 0}$ & \\
\hline
\end{tabular}


uma chave confidencial e individual a todos os profissionais de saúde, que colaboraram na recolha de dados, para aceder à plataforma.

A entrevista aos doentes foi realizada por profissionais de saúde que pelo seu código de conduta profissional garantem o princípio do sigilo, foi pedida a leitura e assinatura da declaração de consentimento informado escrita, na qual estava apresentado sumariamente o protocolo do projeto, as condições de recolha de dados, a garantia da inexistência de mecanismo de coação, a liberdade de desistir do estudo ou de remover a informação recolhida à qual está associada, garantir o anonimato. O tempo médio de entrevista foi de 20 minutos.

\section{DISCUSSÃO}

Ao analisar os dados recolhidos, apesar do instrumento em construção assumir as dimensões de análise definidas segundo a CIF, considerou-se relevante conhecer as dimensões percecionadas pela população adulta em estudo (doentes crónicos). Para o efeito, realizou-se uma análise de componentes principais, com o objetivo de analisar a variância total de cada um dos itens do questionário num conjunto de itens.

Comparando as dimensões teóricas da CIF, com as dimensões identificadas na análise de componentes principais (ACP), verificou-se que, para a população adulta com doença crónica, os itens da lista de atividades e participação, podem ser agrupados em cinco dimensões.

Para testar qual o modelo que apresenta maior fiabilidade interna $(\alpha)$, foi comparado o valor médio de todos os coeficientes possíveis do tipo consistência interna (split-half) entre as dimensões teóricas da CIF (Tabela 5) e as dimensões resultantes do ACP (Tabela 6).

$\mathrm{Na}$ análise da discriminação dos níveis de funcionalidade em amostras diferentes, verificou-se a igualdade de variâncias pelo teste de Levene e a igualdade de média por recurso ao teste $t$, para as dimensões teóricas e para as dimensões resultantes do ACP (Tabela 6). O teste $t$ testou a hipótese nula de que os valores médios do grupo de doentes sem CIT e do grupo de doentes com CIT são iguais, rejeitando esta hipótese para um nível de significância de $5 \%$. O que significa que este instrumento é sensível às diferenças existentes entre doentes crónicos com incapacidade e doentes crónicos sem incapacidade.

De acordo com a observação e análise do coeficiente $\alpha$ de Cronbach nos dois modelos em estudo (teórico e análise de componentes principais), verificou-se que a tabela nacional da funcionalidade apresenta bons níveis de fiabilidade, exceto no modelo da dimensão teórica 'Interações e relacionamentos interpessoais'. Na análise mais pormenorizada da estatística de coeficiente a de Cronbach, nas amostras de doentes crónicos sem e com CIT verificou-se que a fiabilidade foi, em regra, mais elevada no grupo que tem certificado de incapacidade atribuída. No modelo sugerido pela análise de componentes principais, os valores obtidos de fiabilidade foram mais elevados e a discrepância da consistência entre doentes crónicos com e sem CIT, apesar de continuar a verificar-se, foi menos evidente.

Ao analisar-se a diferença de médias entre o grupo de doentes crónicos sem incapacidade atribuída (sem CIT) e

Tabela 5 - Coeficiente $\alpha$ de Cronbach e teste $t$ para o modelo teórico da CIF

\begin{tabular}{|c|c|c|c|c|c|}
\hline \multirow[t]{2}{*}{ Dimensões teóricas } & \multirow{2}{*}{$\begin{array}{c}\text { a de } \\
\text { Cronbach } \\
\text { global }\end{array}$} & \multirow{2}{*}{$\begin{array}{c}\alpha \text { de } \\
\text { Cronbach } \\
\text { sem ClT }\end{array}$} & \multirow{2}{*}{$\begin{array}{c}\text { a de } \\
\text { Cronbach } \\
\text { com CIT }\end{array}$} & \multicolumn{2}{|c|}{$\begin{array}{l}\text { Teste } t \text { para a } \\
\text { igualdade de médias }\end{array}$} \\
\hline & & & & $p$ & $\neq$ de médias \\
\hline \multirow[t]{2}{*}{ d1 - Aprendizagem e aplicação dos conhecimentos } & Razoável & Razoável & Bom & & \\
\hline & 0,67 & 0,67 & 0,82 & 0,000 & $-0,75$ \\
\hline \multirow[t]{2}{*}{ d2 - Tarefas e exigências gerais } & Bom & Razoável & Bom & & \\
\hline & 0,83 & 0,79 & 0,81 & 0,001 & $-2,00$ \\
\hline \multirow[t]{2}{*}{ d3 - Comunicação } & Bom & Razoável & Bom & & \\
\hline & 0,82 & 0,77 & 0,85 & 0,000 & $-1,44$ \\
\hline \multirow[t]{2}{*}{ d4 - Mobilidade } & Excelente & Bom & Excelente & & \\
\hline & 0,92 & 0,89 & 0,90 & 0,000 & $-8,25$ \\
\hline \multirow[t]{2}{*}{ d5 - Autocuidados } & Excelente & Razoável & Excelente & & \\
\hline & 0,93 & 0,77 & 0,93 & 0,000 & $-4,25$ \\
\hline \multirow[t]{2}{*}{ d6 - Vida doméstica } & Bom & Bom & Bom & & \\
\hline & 0,89 & 0,86 & 0,87 & 0,000 & $-4,83$ \\
\hline \multirow[t]{2}{*}{ d7 - Interações e relacionamentos interpessoais } & Fraco & Fraco & Fraco & & \\
\hline & 0,64 & 0,63 & 0,64 & 0,000 & $-1,29$ \\
\hline \multirow[t]{2}{*}{ d8 - Principais áreas da vida } & Bom & Bom & Bom & & \\
\hline & 0,89 & 0,89 & 0,89 & 0,000 & $-2,52$ \\
\hline \multirow[t]{2}{*}{ d9 - Vida comunitária, social e cívica } & Bom & Bom & Bom & & \\
\hline & 0,843 & 0,84 & 0,82 & 0,000 & $-1,19$ \\
\hline
\end{tabular}


Tabela 6 - Coeficiente $\alpha$ de Cronbach e teste $t$ para o modelo da ACP

\begin{tabular}{|c|c|c|c|c|c|}
\hline \multirow{2}{*}{ Dimensões da ACP } & \multirow{2}{*}{$\begin{array}{c}\alpha \text { de } \\
\text { Cronbach } \\
\text { global }\end{array}$} & \multirow{2}{*}{$\begin{array}{c}\text { a de } \\
\text { Cronbach } \\
\text { sem ClT }\end{array}$} & \multirow{2}{*}{$\begin{array}{c}a \text { de } \\
\text { Cronbach } \\
\text { com CIT }\end{array}$} & \multicolumn{2}{|c|}{$\begin{array}{c}\text { Teste } t \text { para a } \\
\text { igualdade de médias }\end{array}$} \\
\hline & & & & $p$ & $\neq$ de médias \\
\hline \multirow[t]{2}{*}{ F1 - Mobilidade e autocuidado } & Excelente & Excelente & Excelente & & \\
\hline & 0,95 & 0,93 & 0,95 & 0,000 & $-10,97$ \\
\hline \multirow[t]{2}{*}{ F2 - Competências gerais } & Excelente & Bom & Excelente & & \\
\hline & 0,90 & 0,88 & 0,9 & 0,001 & $-6,14$ \\
\hline \multirow[t]{2}{*}{ F3 - Competências específicas (cognitivo instrumentais) } & Excelente & Razoável & Excelente & & \\
\hline & 0,90 & 0,79 & 0,93 & 0,000 & $-3,76$ \\
\hline \multirow[t]{2}{*}{ F4 - Sociabilidade } & Bom & Bom & Bom & & \\
\hline & 0,85 & 0,82 & 0,85 & 0,000 & $-2,54$ \\
\hline \multirow[t]{2}{*}{ F5 - Manipulação e manuseio } & Bom & Bom & Bom & & \\
\hline & 0,83 & 0,82 & 0,81 & 0,000 & $-2,38$ \\
\hline
\end{tabular}

o grupo de doentes com incapacidade atribuída (com CIT), verificou-se que em ambos os modelos (teórico e análise de componentes principais), foi rejeitada a igualdade das médias dos dois grupos. Este resultado indica que as diferenças observadas em todas as dimensões foram estatisticamente distintas. É corroborado, assim, o facto de a tabela conseguir distinguir doentes crónicos com incapacidade de doentes crónicos sem incapacidade (pelo menos concorda, em grande medida, com o critério vigente da forma como as pessoas adultas estão divididas em 'sem' e 'com' CIT). Observando globalmente as diferenças das médias nos dois modelos em análise (dimensões teóricas e análise de componentes principais), foram mais significativas as diferenças de médias encontradas no modelo sugerido pela análise de componentes principais.

A análise estatística realizada sugere que os itens, validados para classificar a funcionalidade em doentes crónicos, deverão ser agrupados segundo as dimensões sugeridas pela análise de componentes principais, uma vez que apresentam melhores características psicométricas que as dimensões teóricas descritas na CIF.

Sendo o resultado esperado deste processo, o desenho de uma tabela de funcionalidade para adultos em Portugal, assume-se que os itens que constituem a tabela nacional da funcionalidade serão agrupados e classificados em cinco dimensões: Mobilidade e autocuidado; Competências gerais; Competências específicas; Sociabilidade; Manipulação e manuseio.

Uma vez que a Tabela Nacional de Funcionalidade para as doenças crónicas, em estudo, pode beneficiar de um reagrupamento dos itens em dimensões distintas da CIF, os autores considerarão relevante testar a validade externa da referida tabela, com o objetivo de averiguar se os resultados obtidos com esta amostra podem ser generalizados para outros indivíduos. Para o efeito todos os participantes em estudo responderam concomitantemente à versão portuguesa validada do SF12. Os resultados da correlação dos scores de ambos os testes serão apresentados em artigo próximo.

\section{CONCLUSÃO}

Os autores consideram que a TNF promove um processo inovador, na forma como aborda o estado de saúde dos utentes com doença crónica, porque não é um processo discriminante por patologia crónica, mas discrimina a funcionalidade individual, num período do ciclo de vida associada á cronicidade de uma patologia. Facto que pode ser considerado como uma limitação do estudo.

Para além das limitações inerentes ao carácter inovador do projeto registaram-se, ainda, algumas dificuldades de implementação, como sejam: O envolvimento de um elevado número de colaboradores nas diversas fases do processo; A resistência, por parte dos profissionais de saúde, ao preenchimento dos questionários; A falta de formação dos profissionais de saúde sobre a CIF; A dispersão geográfica dos locais de recolha de dados em todo o território nacional.

Tendo por referência a experiência de outro país ${ }^{20-22}$ considera-se relevante testar a tabela como instrumentos de identificação e quantificação de ganhos de saúde, dos programas de gestão integrada da doença e de unidades prestadoras de cuidados de reabilitação. Sabendo que a tabela nacional de funcionalidade, não substitui, mas complementa, a atual tabela de incapacidade, é relevante averiguar o enquadramento legal da aplicação desta tabela e perceber qual o impacto económico da aplicação da implementação desta medida.

Todavia, concluímos que a TNF apresenta boas características psicométricas, no que diz respeito à consistência, fiabilidade e validade interna, através da validade de conteúdo, validade de constructo, validade convergente e validade discriminante, pelo que se sustenta a premissa da sua aplicação a adultos com doença crónica em Portugal.

\section{AGRADECIMENTOS}

Gostaríamos de agradecer a todos os colaboradores nos diversos procedimentos metodológicos que garantiram a recolha de toda a informação pertinente para construção desta TNF. Painel de peritos, IPO, ANDAR, ANEA, APCL, 
Instituto Gama Pinto; ACES Cávado II; ACES Pinhal Litoral I; ACES da Cova da Beira; ACES Médio Tejo II; ACES Península de Setúbal I; ACES Alentejo Central I; ACES Litoral Alentejano; ACES Algarve II; ACES Algarve III.

\section{PROTECÇÃO DE PESSOAS E ANIMAIS}

Os autores declaram que os procedimentos seguidos estavam de acordo com os regulamentos estabelecidos pelos responsáveis da Comissão de Investigação Clínica e Ética e de acordo com a Declaração de Helsínquia da Associação Médica Mundial.

\section{REFERÊNCIAS}

1. World Health Organization. Assessing national capacity for the prevention and control of noncommunicable diseases: Report of the 2010 Global Survey. Geneva: WHO; 2012.

2. Lopes M, Escoval A, Mendes F, Pereira D, Pereira C, Fonseca C, et al. Violência, abuso, negligência e condição de saúde dos idosos: relatório final. Lisboa: Direção-Geral da Saúde, Universidade de Évora, Universidade NOVA de Lisboa; 2012.

3. Parrish RG. Measuring population health outcomes. Prev Chron Dis. 2010;7:1-11.

4. Organização Mundial da Saúde, Direção-Geral da Saúde. Leitão A (trad/rev). Classificação Internacional de Funcionalidade, Incapacidade e Saúde. Lisboa: DGS, OMS; 2004.

5. Pereira, C. Contributo para a implementação da Classificação Internacional de Funcionalidade, para a identificação de ganhos em saúde nas doenças crónicas. [Dissertação de Mestrado] Lisboa: Instituto Superior das Ciências da Empresa e do Trabalho; 2008.

6. Brach M, Cieza A, Stucki G, Füssl M, Cole A, Ellerin B, et al. ICF Core Sets for breast cancer. J Rehabil Med. 2004;44:S121-7.

7. Cieza A, Stucki A, Geyh S, Berteanu M, Quittan M, Simon A, et al. ICF Core Sets for chronic ischaemic heart disease. J Rehabil Med. 2004;44:S94-9

8. Cieza A, Stucki G, Weigl M, Kullmann L, Stoll T, Kamen L, et al. ICF Core Sets for chronic widespread pain. J Rehabil Med. 2004;44:S63-8.

9. Cieza A, Chatterii S, Andersen C, Cantista P, Herceg M, Melvin J. ICF Core Sets for depression. J Rehabil Med. 2004;44:S128-34.

10. Ruof J, Cieza A, Wolff B, Angst F, Ergeletzis D, Omar Z. ICF Core Sets for diabetes mellitus: J Rehabil Med. 2004;44:S100-6.

11. Kirchberger I, Coenen M, Hierl FX, Dieterle C, Seissler J, Stucki G. Validation of the International Classification of Functioning, Disability and Health (ICF) core set for diabetes mellitus from the patient perspective using focus groups. Diabet Med. 2009;26:700-7.

\section{CONFIDENCIALIDADE DOS DADOS}

Os autores declaram ter seguido os protocolos do seu centro de trabalho acerca da publicação dos dados de doentes.

\section{CONFLITO DE INTERESSES}

Os autores declaram não ter nenhum conflito de interesses relativamente ao presente artigo.

\section{FONTES DE FINANCIAMENTO}

Não existiram fontes externas de financiamento para a realização deste artigo.

12. Mullis R, Barber J, Lewis M, Hay E. ICF core sets for low back pain: do they include what matters to patients? J Rehabil Med. 2007;39:353-7.

13. Stucki A, Daansen P, FuessI M, Cieza A, Huber E, Atkinson R, et al. ICF core sets for obesity. J Rehabil Med. 2004;44:S107-13.

14. Stucki A, Stoll T, Cieza A, Weigl M, Giardini A, Wever D, et al. ICF Core Sets for obstructive pulmonary diseases. J Rehabil Med. 2004;44:S11420.

15. Dreinhöfer K, Stucki G, Ewert T, Huber E, Ebenbichler G, Gutenbrunner C. ICF Core Sets for osteoarthritis. J Rehabil Med. 2004;44:S75-80.

16. Stucki G, Cieza A, Geyh S, Battistella L, Lloyd J, Symmons D. ICF Core Sets for rheumatoid arthritis: J Rehabil Med. 2004;44:S87-93.

17. Cieza A, Schwarzkopf S, Sigl T, Stucki G, Melvin J, Stoll T. ICF Core Sets for osteoporosis, et al. J Rehabil Med. 2004;44:S81-6.

18. Geyh S, Cieza A, Schouten J, Dickson H, Frommelt P, Omar Z, et al. ICF Core Sets for stroke. J Rehabil Med. 2004;44:S135-41.

19. Bosma H, Mheen H, Borsboom G, Mackenbach J. Neighbourhood socioeconomic status and all cause mortality. Am J Epid. 2001;153:363-71.

20. Australian Institute of Health \& Welfare. History of the Internationa classification of Functioning, Disability and Health (ICF): Disability Data Briefing (21); 2002. [consultado 2015 jun 03]. Disponível em: http://www. aihw.gov.au/WorkArea/DownloadAsset.aspx?id=6442475153.

21. Escorpizo R, Finger ME, Glassel A, Gradinger F, Lückenkemper M, Cieza A. A systematic review of functioning in vocational rehabilitation using the International Classification of Functioning, Disability and Health. J Occup Rehab. 2001;21:134-46.

22. Leonardi M, Francescutti C. The "ICF in Italy project" and the activities of the disability Italian Network, meeting of WHO collaborating centres for the family of international classifications. Cologne, Germany 19-25, October; 2003. 